\title{
Erratum zu: Heizungsanlagen
}

\section{Erratum zu: \\ W. Heße, Energieeffiziente Wärmeversorgung von Gebäuden, https://doi.org/10.1007/978-3-658-27571-6_5}

In Kapitel 5 wurden die Gleichungen 5.9 und 5.10 wie folgt korrigiert.

Die zuerst veröffentlichte Gleichung 5.9

$$
\vartheta_{V}=\frac{\vartheta_{i}-e^{x} \times\left(\vartheta_{V, N}-\vartheta_{R, N}\right) \times \varphi+\vartheta_{i}}{1-e^{x}}
$$

wurde korrigiert und lautet nun:

$$
\vartheta_{V}=\frac{e^{x} \times\left[\left(\vartheta_{V, N}-\vartheta_{R, N}\right) \times \varphi+\vartheta_{i}\right]-\vartheta_{i}}{e^{x}-1}
$$

Die zuerst veröffentlichte Gleichung 5.10

$$
x=\frac{\varphi^{p / 1-p} \times\left(\vartheta_{V, N}-\vartheta_{R, N}\right)}{\Delta \vartheta_{m, N}}
$$

wurde korrigiert und lautet nun:

$$
x=\frac{\varphi^{p /(1+p)} \times\left(\vartheta_{V, N}-\vartheta_{R, N}\right)}{\Delta \vartheta_{m, N}}
$$

Die aktualisierte Version des Kapitels finden Sie unter https://doi.org/10.1007/978-3-658-27571-6_5 